\title{
Response elimination durability: A discrete-trial assessment procedure with pigeons
}

\author{
B. KENT PARKER, J. WADE YARBROUGH, and R. REED HARDY \\ West Virginia University, Morgantown, West Virginia 26506
}

\begin{abstract}
Pigeons were trained to respond with equal variable-interval reinforcement schedules in the presence of a green and a red key. They were then trained to not respond by extinguishing responding in the presence of one stimulus and reinforcing not responding in the presence of the other. Responding declined sharply in both components but did not differ systematically in speed of elimination or terminal response rate. Subsequently, response elimination durability was assessed using a new method that minimized the discriminative properties of reinforcement. Four-second red or green negative automaintenance trials were programmed on a new response key, but the original response elimination key continued to present continuously lighted red or green stimuli. Responses to the continuously lighted key had no programmed consequences, and no responses occurred. Substantially fewer responses occurred to negative automaintenance trials if either key presented the stimulus previously associated with reinforcement for not responding, as compared with the stimulus previously associated with extinction.
\end{abstract}

Much attention has recently been directed toward operant procedures for eliminating behavior with positive reinforcement (e.g., Flory, Smith, \& Ellis, 1977; Lowry \& Lachter, 1977; Zeiler, 1976, 1977). Experimental interest has focused on comparing differential reinforcement of other behavior (DRO), a schedule in which food delivery is dependent on not emitting a specific response for a specified period of time, with extinction (EXT) in terms of the speed and durability of response elimination.

Several procedures have been used to assess the durability of response cession produced by DRO and EXT. The most popular has been a variable-time (VT) schedule of response-independent reinforcers following the elimination of baseline responding maintained by variable-interval (VI) reinforcement with DRO or EXT for separate groups of subjects. The rationale for this procedure is that the VT schedule resembles baseline training and might induce responding, even though response resumption is not required for reinforcers to occur, thereby utilizing discriminative but not contingency effects to assess durability. Studies utilizing the VT durability test have unanimously shown greater resumption of responding in EXT than in DRO groups (Harman, 1973; Topping \& Larmi, 1973; Topping, Pickering, \& Jackson, 1971; Uhl, 1973, 1974; Uhl \& Garcia, 1969; Uhl \& Homer, 1974).

A period of EXT following response elimination has also been used to measure recovery of eliminated behavior under nonreinforcement conditions. In contrast with findings using the VT durability test, experi-

Reprints may be obtained from B. Kent Parker, Department of Psychology, West Virginia University, Morgantown, West Virginia 26506. R. R. Hardy is now at the Department of Psychology, St. Norbert College, DePere, Wisconsin 54304. ments employing the EXT test have found higher rates of responding for DRO than for EXT subjects (Pacitti \& Smith, 1977; Uhl, 1974; Uhl \& Garcia, 1969). However, two recent studies employing within-subjects elimination procedures (Lowry \& Lachter, 1977; Mulick, Leitenberg, \& Rawson, 1976) found no differential recovery during an EXT test.

Reconditioning has provided an additional means of measuring response elimination durability. In this procedure, the original baseline schedule of dependent reinforcement is reinstated following the elimination phase. Uhl and his associates (Uhl, 1974; Uhl \& Garcia, 1969) reinstated a VI $30-\mathrm{sec}$ schedule after elimination and found relatively more responding by EXT than by DRO subjects, but the difference was not large. Similarly, Pacitti and Smith (1977) reinstated a VI 30-sec schedule for a 1-h session following response elimination and found more responding by EXT than by DRO subjects.

Finally, a spontaneous recovery procedure has been used to assess the durability of not responding in two investigations employing within-subjects elimination procedures. Zeiler (1971) used a two-component multiple schedule in which DRO and EXT were correlated with different stimuli following original training on a fixed-ratio (FR) schedule. To test for durability during response elimination, the intersession interval was increased from 24 to $72 \mathrm{~h}$. All subjects increased their response rate during the EXT, but not during the DRO component following the 72-h interval. Topping and Ford (1974) also found spontaneous recovery in the EXT but not in the DRO component of a multiple schedule following intersession intervals of 10,25 , and 55 days.

A number of researchers (Mulick et al., 1976; Rescorla \& Skucy, 1969; Uhl \& Garcia, 1969) have noted that the 
reinforcing event may acquire discriminative properties that contribute differentially to the assessed durability of DRO and EXT. Both VT and the reconditioning test of durability favor more responding following EXT than following DRO because stimulus control of responding established during baseline is lost during DRO when reinforcement continues to occur and is correlated with not responding. In contrast, when responding is eliminated by EXT, reinforcement does not occur, and discriminative properties are unchanged when reinforcers are reintroduced during durability testing. Consequently, during the VT and reconditioning tests, discriminative control of responding by reinforcers is weakened in the DRO more than in the EXT conditions.

On the other hand, when durability is assessed with an EXT test condition, the discriminative stimulus properties of reinforcement favor higher rates of responding for DRO than for EXT. The absence of reinforcement during the EXT test for DRO conditions is a change in occurrence of reinforcement and could result in an increase in responding, but an increase in responding following EXT would not be expected, since no change in stimulus conditions occurs.

The purpose of the present experiment was to assess the durability of response elimination with a condition that minimizes the discriminative properties of reinforcement and provides a more extended test of durability than does spontaneous recovery. Accordingly, a procedure similar to Wessells' (1973) discrete-trial retardation test was used, in which the difficulty of conditioning a peck to a former CS- was assessed by pairing the CSwith food in autoshaping trials. A 15-session negative automaintenance procedure (Williams \& Williams, 1969) was administered following response elimination, with DRO and EXT conditions each correlated with a different discriminative stimulus in a two-component multiple schedule. Negative automaintenance trials incorporating 4-sec red or green stimuli were presented on a previously darkened right response key according to a VT 30-sec schedule. Following each trial, an intertrial interval occurred, during which the key was darkened. Thus, a new response key presenting a condition known to induce keypecking was used to assess the durability of not responding to stimuli previously correlated with DRO or EXT conditions and the discriminative properties of reinforcement were minimized when food and trial stimulus presentations were separated by an intertrial interval. Uhl and Garcia (1969) have shown that the discriminative effects of reinforcement are eliminated by a time-out interposed between reinforcers and the next opportunity to respond.

\section{METHOD}

\section{Subjects}

Three adult experimentally naive White King pigeons were maintained at $80 \%$ of their free-feeding weights throughout the experiment. The birds were housed in individual cages, in which they had access to grit and water.

\section{Apparatus}

The experimental chamber was a two-key unit measuring $34 \mathrm{~cm}$ long, $28 \mathrm{~cm}$ high, and $28 \mathrm{~cm}$ wide. The response keys were $2.5 \mathrm{~cm}$ in diameter and $22.5 \mathrm{~cm}$ from the floor. Industrial Electronics Engineers display cells projected stimuli onto the keys from behind. Centered between the keys, $8 \mathrm{~cm}$ from the bottom of the panel, was a $5 \times 5 \mathrm{~cm}$ opening that allowed access to a solenoid-operated grain hopper. White noise was continuously present in the chamber to mask extraneous sounds. The chamber was diffusely lighted from above by a $15-\mathrm{W}$ bulb. Electromechanical scheduling and recording equipment was located in an adjacent room.

\section{Procedure}

Baseline. All birds were shaped to peck the left response key by the method of successive approximations. Each reinforcer was always $3.5 \mathrm{sec}$ access to mixed grain, during which the hopper light was illuminated. Baseline training consisted of random red and green transillumination of the left response key (the right key was uncovered but darkened during baseline and response elimination phases). For the first 2 days of baseline, a VI 30-sec schedule was in effect during the presentations of both red and green stimuli. Thereafter, the prevailing schedule was mult VI $60 \mathrm{sec} /$ VI $60 \mathrm{sec}$. The stimulus presentations were $120 \mathrm{sec}$ in duration and separated by a 10-sec blackout. A daily session consisted of 20 presentations of each stimulus. Baseline conditions were maintained for 15 daily sessions.

Response elimination. Stimuli were presented as in baseline, but the schedule correlated with each was changed. During one color (red for Birds 1 and 3, green for Bird 6), food was no longer available and keypecks had no scheduled consequences (EXT). During the other color, a VT 30-sec tape puller ran continuously and, in the absence of responding, presented food as scheduled. Each response started or reset a $10-\mathrm{sec}$ recycling timer. While the timer operated, it stopped the tape puller, thereby postponing food presentations (DRO). These conditions were maintained for 25 sessions. Following Sessions 5, 10, and 18 , a 72-h intersession interval was initiated, during which no training was given. A $23-\mathrm{h}$ intersession interval separated all other sessions.

Durability test. During this phase, red and green stimuli continued to be randomly presented on the left key, but the duration was reduced to $60 \mathrm{sec}$. Responses on the left key had no programmed consequences. On the right key, negative automaintenance trials were programmed concurrently on a VT $30-\mathrm{sec}$ schedule. A trial consisted of the forward pairing of a 4-sec red or green key light and the magazine operation. If the bird did not peck the trial stimulus, the food hopper was presented for $3.5 \mathrm{sec}$ and the intertrial interval was initiated. When the bird pecked the key light, the trial was immediately terminated, food presentation was cancelled, and the next intertrial interval was initiated. The response key was darkened during all intertrial intervals. Thus, continuous red or green stimuli were randomly programmed on the left or continuous key, and either a red or a green 4-sec trial stimulus was presented on the right, or signal, key for a given 60 -sec period.

\section{RESULTS}

\section{Response Elimination}

Changes in the response rate of each subject across the 23 sessions of response elimination are shown in Figure 1. Responding declined sharply in both conditions over the first four sessions. By the fifth session and thereafter, the rate in both conditions was less than 10 responses/min, with the exception of Sessions 19 and 20 for Bird 1. For Birds 1 and 6, response rates in the DRO component did not differ systematically from 


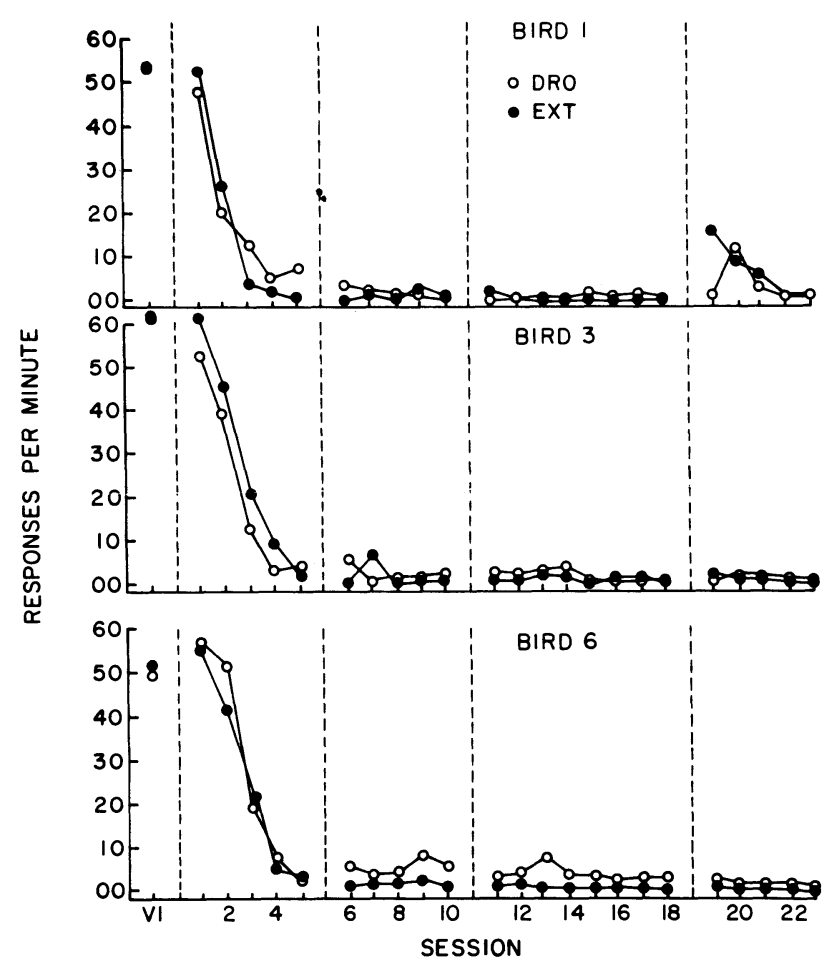

Figure 1. Responses per minute on the last day of VI 60-sec baseline training and in the DRO and EXT components for each of the 23 response elimination sessions. The vertical dashed lines appear between sessions separated by $72 \mathrm{~h}$.

those in the EXT component in either speed of response elimination or terminal rate. For Bird 3, the DRO schedule controlled a lower rate than EXT for the first four sessions. Thereafter, differences in response rates between the DRO and EXT components were not orderly. Little evidence of spontaneous recovery was obtained following the 72 -h intersession intervals after Sessions 5, 10, and 18. Only Bird 1 showed an increased rate of responding during the EXT component following Session 18.

\section{Durability Test}

Although both keys were uncovered and lighted at various times during this phase, none of the birds responded on the continuous key. Therefore, the data presented are based on pecks to the signal key.

The percentage of trials with a response was calculated as a function of the stimuli present on the continuous and signal key for each daily session. Since stimuli were programmed independently on the two keys, four different combinations of DRO and EXT stimuli were possible. Figure 2 summarizes these data for each subject and shows the group mean. The left panel of each graph shows responding when the DRO stimulus was on the continuous key; the right panel shows responding during the EXT stimulus.

Examination of both individual and group data indicates that responding to the signal key was a joint function of the signal and continuous key stimuli. The presence of the stimulus previously associated with the
DRO schedule on either the continuous or signal key decreased the percentage of trials with a peck. With the DRO stimulus on the continuous key (left panels), the percent of trials with a peck was substantially lower with the DRO stimulus than with the EXT stimulus on the signal key. This is most clearly shown for Bird 6 and also for the group mean across all 15 sessions. Birds 1 and 3 responded on a lower percentage of DRO than of EXT trials on 9 of 15 and 13 of 15 sessions, respectively. With the EXT stimulus on the continuous key (right panels), the percentage of trials was also lower in the presence of the DRO stimulus than in the presence of the EXT stimulus on the signal key. Bird 3 showed a lower percentage across all sessions, and Birds 1 and 6 showed a lower percentage on 11 of 15 and 12 of 15 sessions, respectively.

Differential control by continuous key stimuli was of a lesser magnitude than that of the signal key stimuli, but it can be seen by comparing left and right panels in Figure 2. In general, more trials with a peck occurred with the EXT stimulus on the continuous key than with the DRO stimulus. In terms of the group data, with the DRO stimulus on the signal key, each of the data points for the continuous EXT stimulus (right panels) was

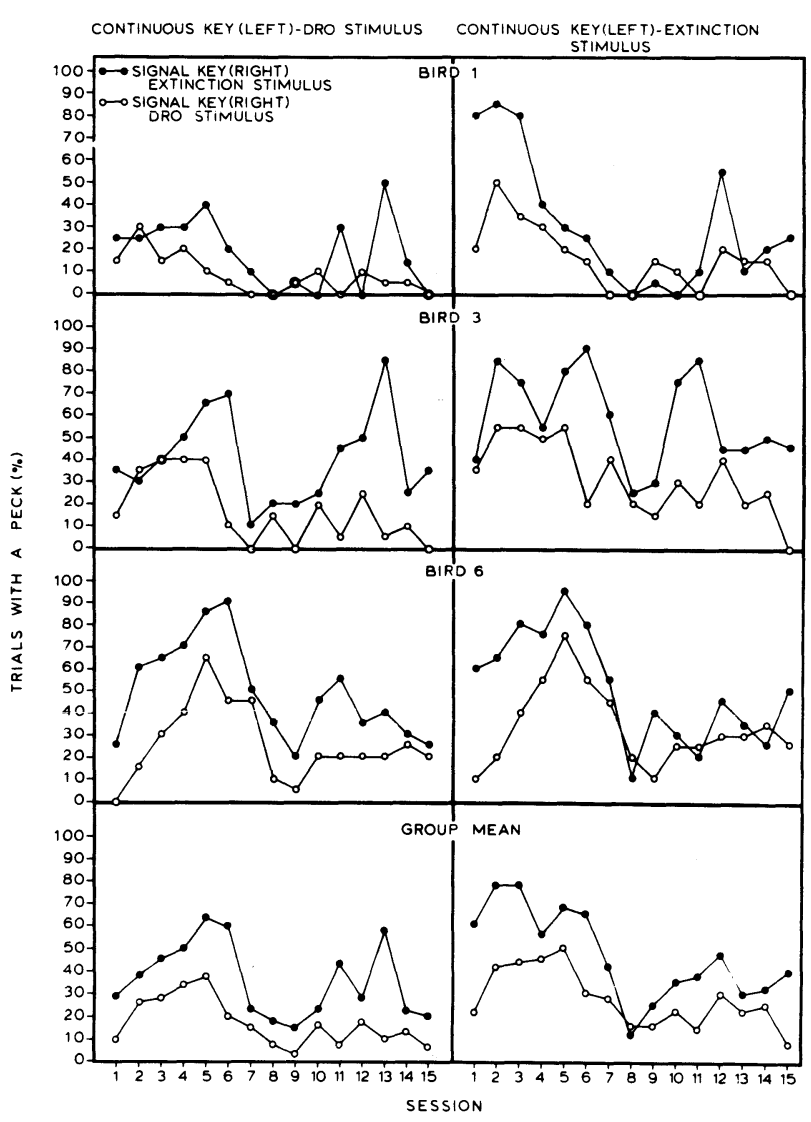

Figure 2. Percent trials with at least one peck for each subject and the group average as a function of the DRO stimulus present on the continuous key (left panels) and the EXT stimulus present on the continuous key (right panels). Filled circles are for the EXT stimulus on the signal key; open circles are for the DRO stimulus on the signal key. 
higher than the corresponding session for the continuous DRO stimulus (left panels). With the EXT stimulus on the signal key, 12 of 15 data points for the continuous EXT stimulus were higher than the data points for the continous DRO. The data of individual subjects were consistent with the group data in terms of showing a preponderance of higher right-panel data points than left-panel data points.

\section{DISCUSSION}

The major finding of the present experiment was that DRO produces greater relative permanence of response elimination than does EXT using a new procedure for assessing response elimination durability. The probability of a peck on a negative automaintenance trial was reduced if the trial incorporated a stimulus previously associated with a DRO rather than an EXT schedule. Moreover, the probability of a peck on the automaintenance trial was also reduced if a concurrently programmed response key displayed the DRO rather than the EXT stimulus.

These findings support the results of the majority of previous durability studies in showing greater permanency of effect for DRO compared with EXT. More important, by using the discretetrial procedure to assess durability, the discriminative properties of reinforcement, which confound VT, EXT, and reconditioning durability tests, were minimized by the intertrial intervals. Uhl and Garcia (1969) investigated a condition designed to minimize discriminative effects of reinforcement in response elimination schedules of DRO and EXT and a condition designed to maximize these effects. For DRO subjects, the condition designed to minimize discriminative effects separated reinforcement produced by meeting the DRO requirement (cessation of responding for $30 \mathrm{sec}$ ) from the next opportunity to respond with a $27-\mathrm{sec}$ time-out, during which the lever was retracted and all lights were turned out. In the maximize condition, DRO subjects received a second reinforcer at the end of the time-out, immediately before the next opportunity to respond. The EXT subjects received exactly the same response elimination procedures, except that they did not receive reinforcement at the beginning of the time-out. Not responding for $30 \mathrm{sec}$ produced the time-out, and a reinforcer occurred at the end of the time-out only for subjects in the maximize condition. The results showed considerably slower response elimination for the maximize condition, indicating that the time-out was effective in decreasing the discriminative effects of reinforcement. Similarly, the present durability test separated the opportunity to respond to a trial stimulus from the preceding food presentation with a 15 - to 45 -sec intertrial interval, during which the signal key was darkened.

Although Zeiler (1971) found that DRO eliminated responding more rapidly than EXT in a multiple-schedule design, the results of our elimination phase were consistent with those of Lowry and Lachter (1977) and Topping and Ford (1975) in showing that DRO was no more effective in eliminating responding than was EXT in the majority of subjects. This difference might be accounted for by Zeiler's (1971) use of either relatively shorter DRO values or FR schedules during baseline, or both.

The absence of spontaneous recovery for the EXT condition following the 72-h intersession interval is inconsistent with the findings of both Zeiler (1971) and Topping and Ford (1974). A number of variables could be responsible for this discrepancy, but it is noteworthy that Zeiler (1971) used FR schedules during baseline and longer duration stimulus components $(5 \mathrm{~min}$, compared with $2 \mathrm{~min}$ in the present experiment), whereas Topping and Ford's minimum intersession interval was $240 \mathrm{~h}$. Despite failure of the present experiment to replicate the spontaneous recovery effect, the results from the negative automaintenance test clearly indicate that DRO produces greater response elimination durability than does EXT.

\section{REFERENCES}

Flory, R. K., Sмith, E. L. P., \& Ellis, B. B. The effects of two response-elimination procedures on reinforced and induced aggression. Journal of the Experimental Analysis of Behavior, $1977,27,5-15$.

Harman, R. E. Response elimination in concurrent and single operant situations with pigeons. Learning and Motivation, 1973, 4, 417-431.

Lowry, M. A., \& Lachter, G. D. Response elimination: A comparison of four procedures. Learning and Motivation, 1977, 8, 69-76.

Mulick, J. A., Leitenberg, H., \& Rawson, R. A. Alternative response training, differential reinforcement of other behavior, and extinction in squirrel monkeys (Saimiri sciureus). Journal of the Experimental Analysis of Behavior, 1976, 25, 311-320.

PacitTi, W. A., \& Sмiтh, N. F. A direct comparison of four methods for eliminating a response. Learning and Motivation, 1977, 8, 229-237.

Rescorla, R. A., \& Skucy, J. C. Effect of response-independent reinforcers during extinction. Journal of Comparative and Physiological Psychology, 1969, 67, 381-389.

Topping, J. S., \& ForD, T. W. Response elimination with DRO and extinction: A within-subject comparison. Psychological Record, 1974, 24, 563-568.

Topping, J. S., \& Ford, T. W. A within-subject comparison of three response-elimination procedures in pigeons. Bulletin of the Psychonomic Society, 1975, 6, 257-260.

Topping, J. S., \& Larmi, O. K. Response elimination effectiveness of omission and two extinction training procedures. Psychological Record, 1973, 23, 197-202.

Topping, J. S., Pickering, J. W., \& Jackson, J. A. The differential effects of omission and extinction following DRL pretraining. Psychonomic Science, 1971, 24, 137-138.

UHL, C. N. Eliminating behavior with omission and extinction after varying amounts of training. Animal Learning \& Behavior, 1973, 1, 237-240.

UHL, C. N. Response elimination in rats with schedules of omission training, including yoked and response-independent reinforcement comparisons. Learning and Motivation, 1974, 5, 511-531.

Uhl, C. N., \& Garcia, E. E. Comparison of omission with extinction in response elimination. Journal of Comparative and Psychological Psychology, 1969, 69, 554-562.

Uhl, C. N., \& Homer, A. L. Omission training compared with yoked controls and extinction in multiple schedule discrimination learning. Animal Learning \& Behavior, 1974, 2, 317-324.

Wessells, M. G. Errorless discrimination, autoshaping and conditioned inhibition. Science, 1973, 182, 941-943.

Williams, D. R., \& Williams, H. Auto-maintenance in the pigeon: Sustained pecking despite contingent non-reinforcement. Journal of the Experimental Analysis of Behavior, 1969, 12, 511-520.

ZEILER, M. D. Eliminating behavior with reinforcement. Journal of the Experimental Analysis of Behavior, 1971, 16, 401-405.

ZE ILER, M. D. Positive reinforcement and the elimination of reinforced responses. Journal of the Experimental Analysis of Behavior, 1976, 26, 37-44.

ZEILER, M. D. Elimination of reinforced behavior: Intermittent schedules of not-responding. Journal of the Experimental Analysis of Behavior, 1977, 25, 23-32.

(Received for publication July 14, 1980.) 